\title{
Post Treatment of Guided Wave by using Wavelet Transform in the Presence of a Defect on Surface
}

\author{
MARRAKH Rachid ${ }^{1}$, ALOUANE Houda ${ }^{2}$, BELHOUSSINE DRISSI Taoufiq ${ }^{3}$, NSIRI Benayad ${ }^{4}$, ZAYRIT Soumaya $^{5}$ \\ Laboratory Industrial Engineering, Information Processing and Logistics (GITIL) Faculty of Science \\ Ain Chock University of Hassan II-Casablanca, Morocco ${ }^{1,2,3,5}$ \\ Research Center STIS, M2CS, Higher School of Technical Education of Rabat \\ Mohammed V University in Rabat, Morocco ${ }^{2,4}$
}

\begin{abstract}
This article presents a Lamb wave processing by using two methods: Fast Fourier Transform (FFT2D) and Continuous Wavelet Transform (CWT) using Morlet wavelet. This treatment is done for a structure of two aluminum-copper plates, which are in contact edge to edge of a perpendicular junction with a thickness " $e$ " in the presence of a rectangular and symmetrical defect located on the surface of the junction with a depth "d". The aim under this study is to calculate the transmission and reflection energy coefficients by the two methods. The results of simulation obtained by Comsol software of an incident wave $S_{0}$ at $F=800 \mathrm{kHz}$ indicate us a good coherence between the two methods (FFT2D and CWT).
\end{abstract} \section{FFT2D}

Keywords_Lamb wave; defect; reflection; transmission; CWT;

\section{INTRODUCTION}

Lamb [1] presents dispersion's equation of Lamb waves that propagate in an elastic plate. This discovery would lead to many applications in different areas such as non-destructive testing. Recently, Viktorov [2] will devote a large part of his work to these waves called Lamb waves. Among the advantages of these waves, we find their propagation over a long distance that can attain a few hundred meters depending on the configuration of the structure and the frequency of the transducers used.

The relationship between the time and the displacements is obtained using the resolution of wave Lamb propagation equations.

These displacements are treated by FFT in order to extract dispersion's curves and to convert incident wave into transmitted and reflected waves. Recently, we remark a significant use of wavelet transformation in domain of nondestructive control.

Wavelet analysis was introduced in the early 1980, it gives a representation of the signals allowing the simultaneous enhancement of the temporal and frequency information (timefrequency localization). Thus the need for wavelets, a family of functions deduced from the same function (called mother wavelets) by translation and dilation operation, was felt by noting that the Fourier transform which dominated from the beginning of 19th century, lost during the projection the control of the temporal variable during the projection and is still unable to describe locally (in time or space) the frequency behavior of the signals.
Pablo R Souza and Euripedes G O Nobrega [3] presented a method for locating and assessing the severity of damages in aluminum plates using a Lamb wave approach through a circular array of sensors and a centered actuator.

Xin Zhang et al [4] presented an experimental study on the simulated acoustic emission sources with different propagation distances, types and defect for rail defect detection. Sergio Cantero-Chinchilla et al [5] presented SHM methods for damage detection and localization in plate-like structures relied on signal post-processing techniques applied to ultrasonic guided waves. Fei Gao et al [6] are used the Time of flight based method and the continuous wavelet transform in order to localize the damage in composites laminates.

Kaihong Zheng et al [7] proposed a non-destructive testing and evaluation (NDE) method based on Lamb waves in order to detect damage in stiffened composite panels. Jinrui Zhang et al [8] are employed the CWT to analyze the Lamb wave dispersion of the detected signal. Bo Feng et al [9] are presented a method that is able to detect and assess delamination's length in anisotropic CFRP plates using chirpexcited Lamb wave and wavelet analysis. Faeez A. Masurkara and Nitesh P. Yelveb [10] are carried out on an aluminum plate with and without damage using Lamb wave in order to locate single as well as multiple damage based on wavelet transform algorithm. Michele Carboni et al [11] are studied the propagation of Lamb wave for quasi-isotropic CFRP laminate with the aim to set-up a "single propagation mode" approach. Guoqi Zhao et al [12] are used ultrasonic guided wave in order to detect the delamination of composite double cantilever beams (DCBs). The study of Zhongqing Su et al [13, 14] based on a Lamb wave propagation-based delamination identification scheme for CF/EP composite structures [13] to detect delamination in the structures. Then they are focused on their study to provide a comprehensive on the Lamb wave-based damage identification approaches for composites structures [14].

Among the treatment that used FFT we find, Taoufiq Belhoussine Drissi et al [15] that studied the reflection and the transmission of guided wave at the right junction of two different elastic plates with the presence of a defect, and Mouna Seddiki, Hakim Djelouah [16] which their study focus on the identification of propagative Lamb mode in a plate with the presence of a defect. 
For the treatment used by WT we find, Beata Zima and Magdalena Rucka [17] that presented an experimental study of guided wave propagation on a steel plate with an internal defect by using continuous wavelet processing to obtain accurate reconstruction of reflected waves, and a study proposed by D. Waltisberg, R. Raišutis [18] based on the separation of the first symmetric mode $S_{0}$ and the first asymmetric mode $\mathrm{A}_{0}$ and the reliable estimation of their group velocities by using tree methods among them the wavelet transform. LeiYangI. CharlesUme [19] are used CWT for a thin steel plate in order to calculate the transmission coefficients of laser-generated Lamb waves. Taoufiq Belhoussine Drissi et al [20] are used the wavelet transform for a speech signal in order to determine the choice of the appropriate wavelet analyzer with the method of extraction of MFCC coefficients for an assistance in the diagnosis of Parkinson's disease. Tsun-Yen $\mathrm{Wu}$ [21] et al are used the CWT to help identify wave packets of the $S_{0}$ and $A_{0}$ Lamb wave modes in order to detect defects in thin structures. Lei Yang, I et al [22] are used the LEU technique to inspect the notch depths in thin steel plates and by the CWT they computed the transmission coefficient of Laser generated Lamb wave.

Another research used both the FFT and CWT, among them M. Sifuzzaman [23] that compared analytically the advantages of the wavelet transform according to the Fourier transform and also Mhammed El Allami and Hassan Rhimini [24] that used the wavelet transformation to determine the energies of the different Lamb modes propagating in a steel plate with an internal, rectangular and symmetrical defect with respect to the axis of propagation.

In the present work, we come up with a treatment by the continuous wavelet and Fourier transform (CWT and FFT2D) for two isotropic and thin plates aluminum-copper which are connected to each other by a perpendicular junction with the presence of a rectangular and symmetrical defect on the surface of the junction. The objective of this treatment is to calculate the reflection and transmission energy coefficients, then compare the results obtained by the two methods (FFT2D and CWT).

\section{Presentation OF the Studied Structure}

For this treatment, we use the structure below:

The structure contains two isotropic and thin plates aluminum-copper which are in contact edge to edge with thickness e $=2 \mathrm{~mm}$ in the presence of a rectangular defect with depth $\mathrm{d}$ and located symmetrically on the median plan.

Aluminum is indicated by index 1 and characterized by the density $\rho_{1}$, a longitudinal $c_{11}$ and transversal velocity $c_{t 1}$. Copper is indicated by index 2 and characterized by the density $\rho_{2}$, a longitudinal $c_{12}$ and transversal velocity $c_{12}$

The table below presents the characteristics of aluminum and copper plates:

TABLE. I. CHARACTERISTICS OF ALUMINUM AND COPPER PLATES

\begin{tabular}{|l|l|l|}
\hline Characteristics & Aluminum & Copper \\
\hline Density $\left(\mathrm{kg} / \mathrm{m}^{3}\right)$ & 2799 & 8705 \\
\hline Transversal velocity $(\mathrm{m} / \mathrm{s})$ & 3115 & 2360 \\
\hline Longitudinal velocity $(\mathrm{m} / \mathrm{s})$ & 6320 & 4728 \\
\hline
\end{tabular}

\section{NUMERICAL STUDY}

The study is done by Comsol software, the structure used in Finite Element Method (FEM) is identical to Fig. 1.

The incident mode $\mathrm{S}_{0}$ is emitted from aluminum plate with frequency $F=800 \mathrm{kHz}$. Each plate has a length of $100 \mathrm{~mm}$ with thickness $\mathrm{e}=2 \mathrm{~mm}$.

The processing is done with the aim of studying the reflection and the transmission of Lamb wave through the junction by FFT2D and CWT.

The studied junction is situated at $\mathrm{x}=100 \mathrm{~mm}$, the following Fig. 2 indicate us the presence of incident and reflected wave between 0 and $100 \mathrm{~mm}$ and transmitted wave for a value more than $100 \mathrm{~mm}$.

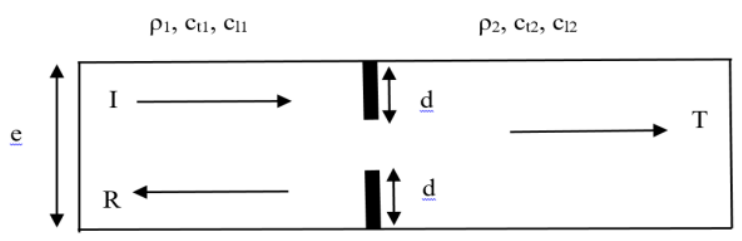

Fig. 1. Structure of Aluminum-Copper Plates with a Defect.

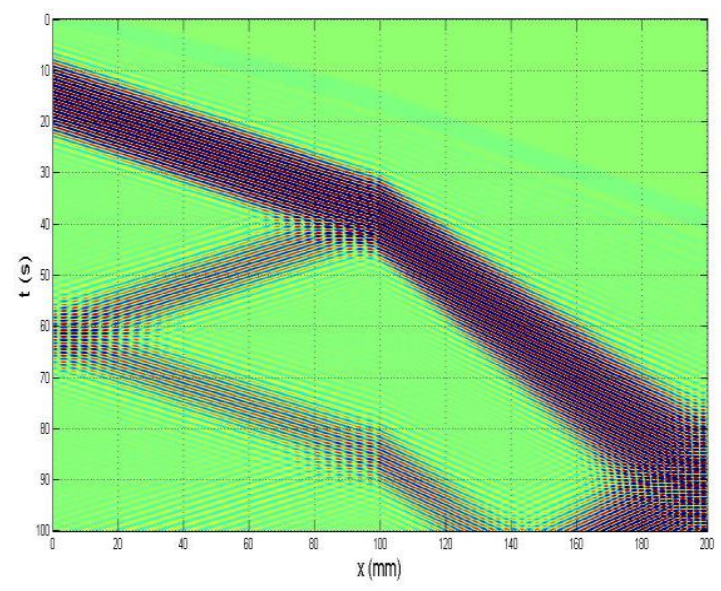

Fig. 2. Time-Position Image of Displacements $(F=800 \mathrm{kHz})$.

\section{TReatment By FFT2D Method}

From the time-position signal, we calculate the temporal Fourier transform of the signal $\mathrm{s}(\mathrm{x}, \mathrm{t})$, we obtained a signal called $\varphi(\mathrm{x}, \mathrm{F})$ for a fixed position. For each frequency $\mathrm{F}$, we apply a second Fourier transform and we obtained the signal $\psi(\mathrm{K}, \mathrm{F})$ (Fig. 1). This signal resulting from this double Fourier Transform is located in the dual frequency-wavenumber space. See Fig. $4(\psi(\mathrm{K}>0, \mathrm{~F})$ incident wave, $\psi(\mathrm{k}<0, \mathrm{~F})$ reflected wave $)$ and Fig $6(\psi(K>0, F)$ transmitted wave $)[25]$.

To identify different modes presented in the simulated structure, we superpose the theoretical dispersion curves on the numerical study.

Then we cut the signal resulting from the double Fourier transform at the frequency $\mathrm{F}=800 \mathrm{kHz}$ to obtain the magnitudes of each mode as shown in Fig. 5 and Fig. 7. 


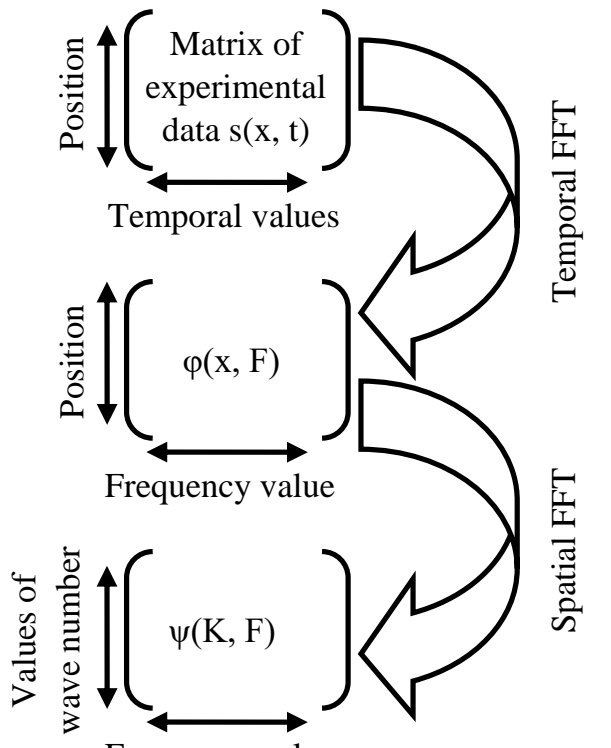

Frequency value

Fig. 3. Signal Processing Applied Spatial and Temporal FFT.

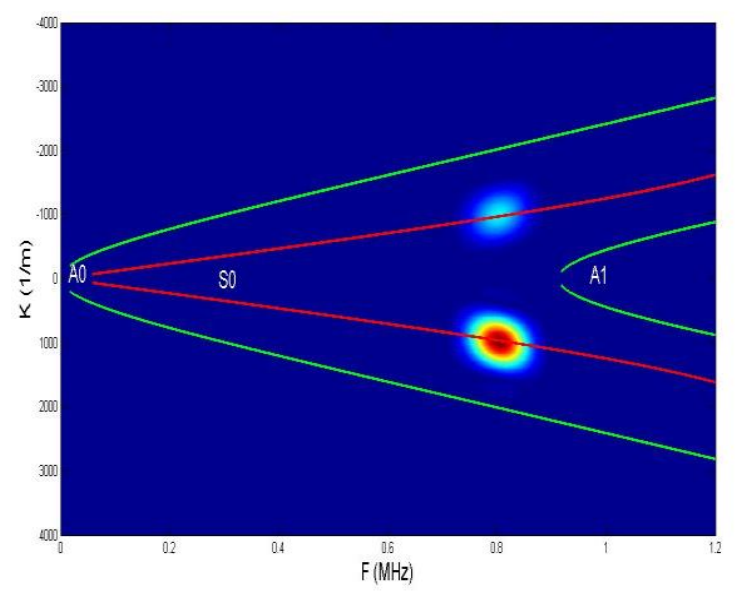

Fig. 4. Representation Frequency-Wave Number for Aluminum Plate.

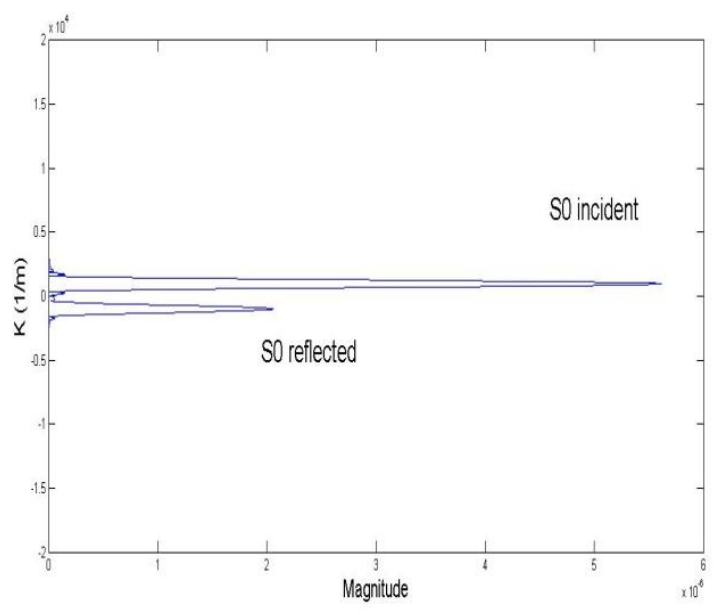

Fig. 5. The Magnitude of the Transmitted Mode.

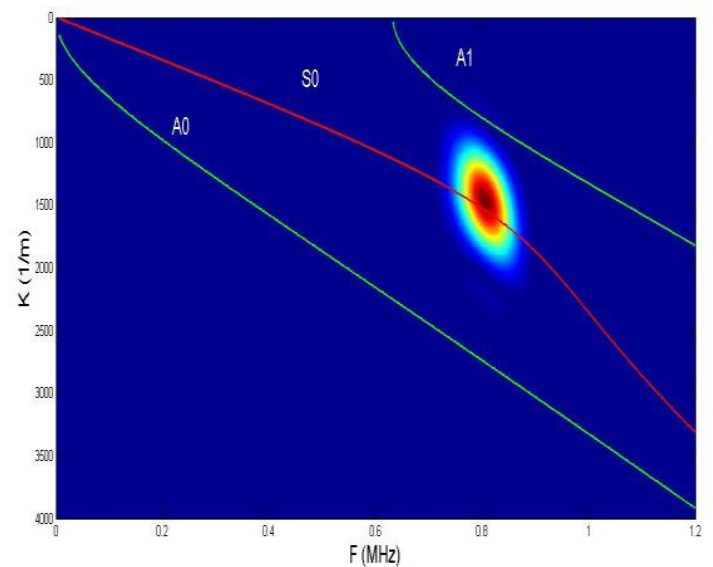

Fig. 6. Representation Frequency-Wave Number for Copper Plate.

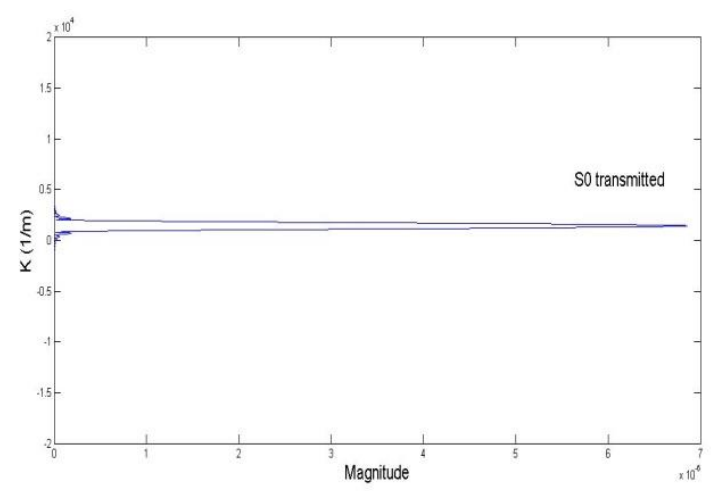

Fig. 7. The Magnitude of the Transmitted Mode.

The distribution of the energy of the incident mode between the reflected and transmitted mode is done by calculating the magnitude of each mode.

The superposition of the theoretical dispersion curves on those obtained by FFT2D show us that at the frequency $\mathrm{F}=$ $800 \mathrm{kHz}$, we have just the symmetrical mode $\mathrm{S}_{0}$ while the junction is symmetrical.

\section{Treatment by the Wavelet Transform Method}

The CWT has been developed to overcome some resolution defaults of the Fourier Transform. It is able to provide a simultaneous time-frequency representation of the signal. In 1982, Morlet [26] opened the way to the solution by building the wavelet transform.

The continuous wavelet transform uses two parameters: one called the scale of the wavelet "a", it can give different version of wavelets by compressing, and dilating the same mother wavelet, it presents the inverse of the frequency. The other is the translation parameter "b" which translates the wavelet along the time axis of the signal. The continuous wavelet transform of a signal $s(t)$ is defined by:

$$
\mathrm{W}(\mathrm{a}, \mathrm{b})=\int_{-\infty}^{+\infty} \psi^{*}\left(\frac{\mathrm{t}-\mathrm{b}}{\mathrm{a}}\right) \mathrm{s}(\mathrm{t}) \mathrm{dt}
$$


Where: $\Psi(\mathrm{t})$ is the wavelet function, $\Psi^{*}(\mathrm{t})$ is $\Psi(\mathrm{t})$ complex conjugate, $\mathrm{W}(\mathrm{a}, \mathrm{b})$ are the continuous WT coefficients.

We apply the continuous wavelet transform by using the mother wavelet Morlet for displacements collected at points situated before the defect $(x=40 \mathrm{~mm})$ and after the defect $(x=$ $110 \mathrm{~mm}$ ) in order to obtain the 3D plot of wavelet coefficients. See Fig. 8 and Fig. 9.

In general, the maximum wavelet coefficient depends on the shape of the wavelet: when the section of the signal has the same shape as the wavelet, we obtain the maximum value of the wavelet coefficients.

In our case, we confirm that $\mathrm{a}=10$ corresponding to the maximum value of coefficients wavelet as shown in Fig. 10 and Fig. 11, then the row of the coefficient matrix for this scale was plotted as function of time. See Fig. 12 and Fig. 13.

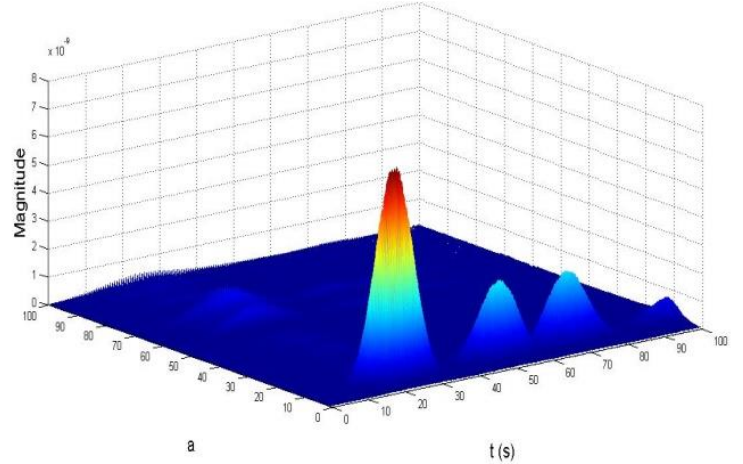

Fig. 8. Wavelet Coefficients for Displacements before the Defect.

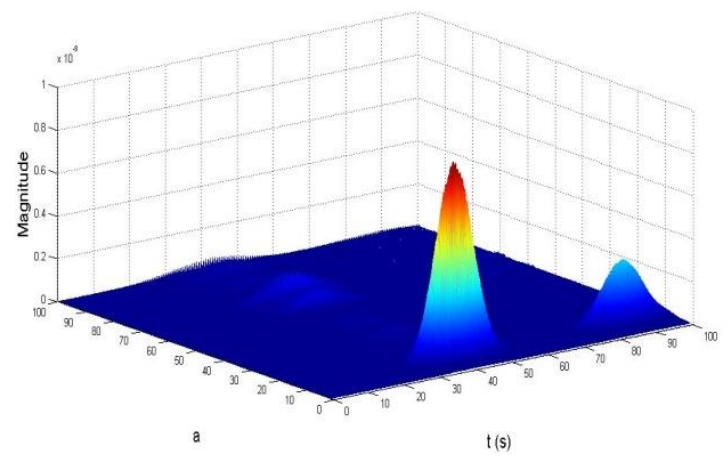

Fig. 9. Wavelet Coefficients for Displacements after the Defect.

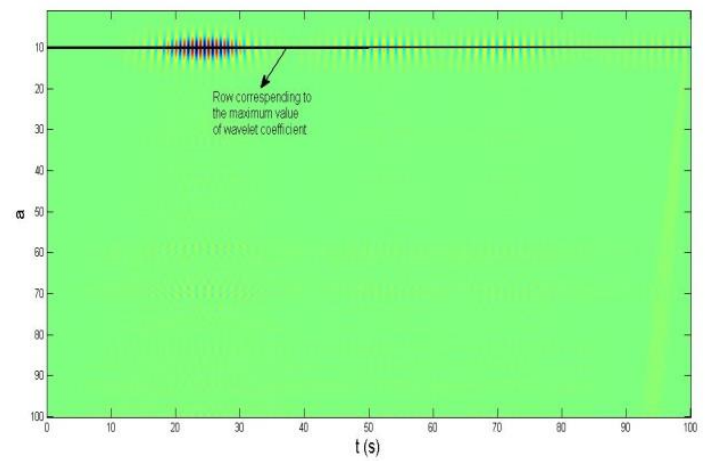

Fig. 10. Time-Scale Representation of Wavelet Coefficients for the Displacements before the Defect at $\mathrm{F}=800 \mathrm{kHz}$.

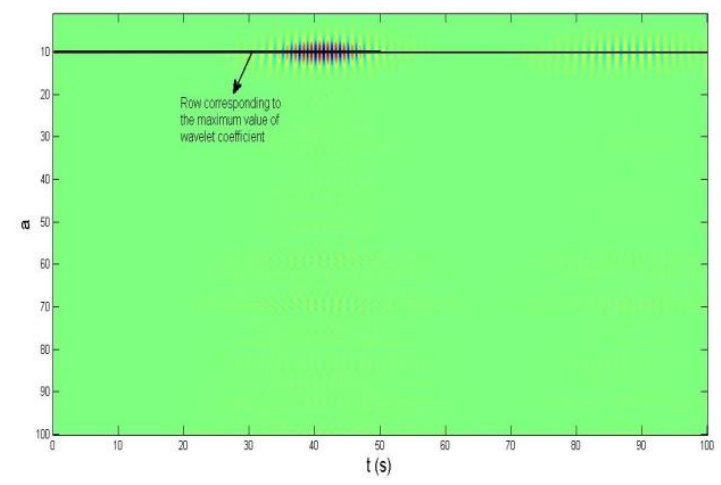

Fig. 11. Time-Scale Representation of Wavelet Coefficients for the Displacements after the Defect at $\mathrm{F}=800 \mathrm{kHz}$.

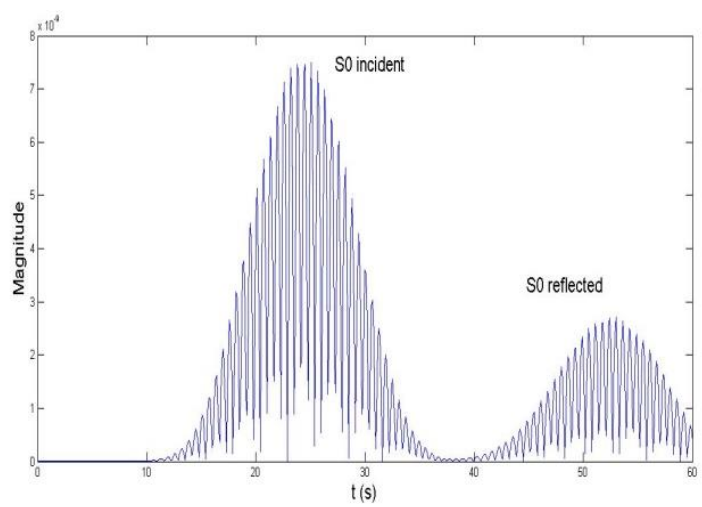

Fig. 12. Wavelet Coefficients for the Displacements: before the Defect at $\mathrm{F}=$ $800 \mathrm{kHz}$.

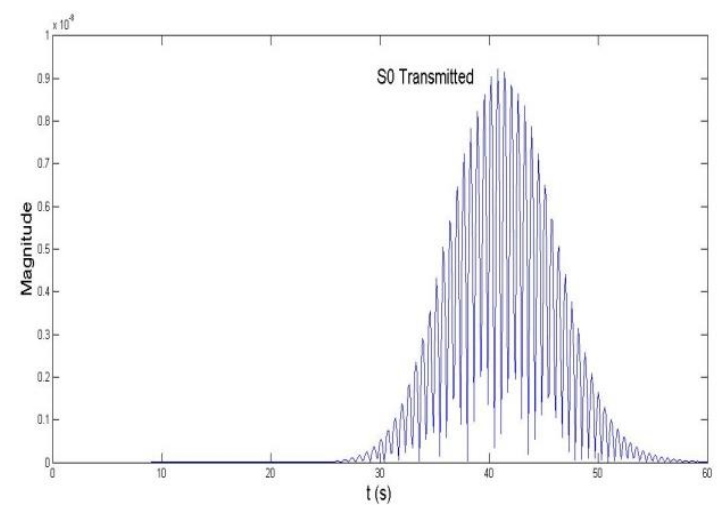

Fig. 13. Wavelet Coefficients for the Displacements after the Defect at $\mathrm{F}=$ $800 \mathrm{kHz}$.

After the determination of the magnitudes of incident, reflected and transmitted mode for each method (Fig. 5, Fig. 7 for the FFT2D and Fig. 12, Fig. 13 for CWT ), now we can deduce the reflection and transmission energy coefficients by applying the following equations [15, 24].

$$
\begin{aligned}
& \mathrm{R}\left(\mathrm{S}_{\mathrm{i}}\right)=\frac{\left(\mathrm{A}^{\mathrm{R}}\left(\mathrm{S}_{\mathrm{i}}\right) * \zeta^{\mathrm{I}}\left(\mathrm{S}_{0}\right)\right)^{2}}{\left(\mathrm{~A}^{\mathrm{I}}\left(\mathrm{S}_{0}\right) * \zeta^{\mathrm{R}}\left(\mathrm{S}_{\mathrm{i}}\right)\right)^{2}} \\
& \mathrm{~T}\left(\mathrm{~S}_{\mathrm{i}}\right)=\frac{\left(\mathrm{A}^{\mathrm{T}}\left(\mathrm{S}_{\mathrm{i}}\right) * \zeta^{\mathrm{I}}\left(\mathrm{S}_{0}\right)\right)^{2}}{\left(\mathrm{~A}^{\mathrm{I}}\left(\mathrm{S}_{0}\right) * \zeta^{\mathrm{T}}\left(\mathrm{S}_{\mathrm{i}}\right)\right)^{2}}
\end{aligned}
$$


Where: $\mathrm{A}^{\mathrm{I}}, \mathrm{A}^{\mathrm{R}}$ and $\mathrm{A}^{\mathrm{T}}$ are the magnitudes of the modes where the higher index present respectively incident, reflected and transmitted mode. The coefficients $\zeta$ are defined by the module of the normal displacement on the surface of the plate divided by the square root of Poynting vector [11].

$\zeta=\frac{\left|\mathrm{U}_{\mathrm{z}}\left(\mathrm{z}= \pm \frac{\mathrm{e}}{2}\right)\right|}{\sqrt{\phi}}$

\section{Results OF THE Two PROCESSING}

The transmission and reflection energy coefficients obtained by the two methods (FFT2D and CWT) in the case of the incident mode is the symmetrical mode $\mathrm{S} 0$ at frequency $\mathrm{F}=$ $800 \mathrm{kHz}$ in the presence of a defect, where the report of the depth of defect by the thickness of the plate is $(\mathrm{d} / \mathrm{e}=1 \%, 2 \%$, $3 \%, 4 \%$ and 5\%) are presented in the following Fig. 14:

The continuous curve in blue indicate the reflection energy coefficient obtained by FFT2D and the curve with stars is the reflection energy coefficient obtained by CWT.

The continuous curve in red indicate the transmission energy coefficient obtained by FFT2D and the curve with stars is the reflection energy coefficient obtained by CWT.

The continuous curve in black indicate the energy conservation $\mathrm{R}+\mathrm{T}$ obtained by FFT2d and the curve with stars is the energy conservation obtained by CWT.

This figure indicates that the results of the two methods in presence of a defect with $(\mathrm{d} / \mathrm{e}=1 \%, 2 \%, 3 \%, 4 \%$ and $5 \%)$ are identical with a neglected error, and an energy conservation $\mathrm{R}+\mathrm{T} \approx 1$. We can deduce that there is a good coherence between FFT2D and CWT and when the report d/e increase the reflection energy coefficient increase and the transmission energy coefficient decrease.

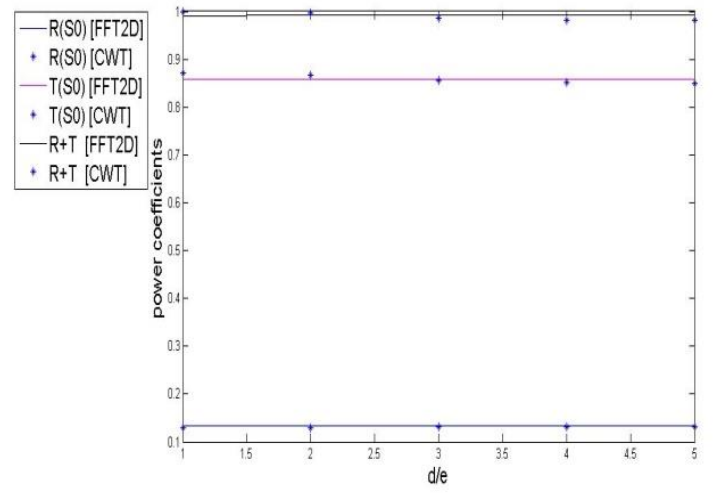

Fig. 14. Transmission and Reflection Energy Coefficients and Energy Conservation.

\section{CONCLUSION}

In this paper, we proposed a treatment of Lamb wave for a two aluminum-copper plates, which are in contact edge to edge by a perpendicular junction with an internal, rectangular and symmetrical defect. This processing is done by two methods: the Continuous Wavelet Transform (CWT) and the Fast Fourier Transform (FFT2D) in order to calculate the reflection and transmission energy coefficients. The obtained results showed us a good coherence between the two methods (FFT2D and CWT). By increasing the thickness of the defect, the reflection energy coefficient increased and the transmission energy coefficient decreased. As prospect, we can use other mother wavelet such us Coifman, Daubechies etc.

\section{REFERENCES}

[1] H. Lamb, "On waves in an elastic plate," Proc. Roy. Soc. of London, 1917, pp.114-128.

[2] I.A. Viktorov, "Rayleigh and Lamb waves," Plenum Press, New York, 1967.

[3] Pablo R Souza. Euripedes G O Nobrega, "A Lamb Wave Based Method for the Assessment of Faults in Aluminium Plates" 8th IFAC Symposium on Fault Detection, August 29-31, 2012. Mexico City, Mexico.

[4] Xin Zhang, Naizhang Feng, Yan Wang, Yi Shen, "An analysis of the simulated acoustic emission sources with different propagation distances, types and depths for rail defect detection", Applied Acoustics, 86 pp 80-88, 2014.

[5] Sergio Cantero-Chinchillaa,d, Juan Chiachíob, Manuel Chiachíoc, Dimitrios Chronopoulosa, Arthur Jonesa, "A robust Bayesian methodology for damage localization in plate-like structures using ultrasonic guided-waves", Mechanical Systems and Signal Processing 122, pp192-205, 2019.

[6] Fei Gao, Liang Zeng, Jing Lin,, Yongsheng Shao, "Damage assessment in composite laminates via broadband Lamb wave", Ultrasonics, vol. 86, 49-58, 2018.

[7] Kaihong Zheng, Zheng Li, Zhaoyang Ma, Jianlin Chen, Jie Zhou, Xianyue $\mathrm{Su}$, "Damage detection method based on Lamb waves for stiffened composite panels", Composite Structures, vol. 225, 2019.

[8] Jinrui Zhang, Hongyan Ma, Wangji Yan, Zongjin Li, “ Defect detection and location in switch rails by acoustic emission and Lamb wave analysis: A feasibility study", Applied Acoustics, vol. 105, 67-74, 2016.

[9] Bo Feng, Artur Lopes Ribeiro, Helena Geirinhas Ramos, "A new method to detect delamination in composites using chirp-excited Lamb wave and wavelet analysis", NDT and E International, vol. $100,64-73$, 2018.

[10] Faeez A. Masurkara, Nitesh P. Yelveb, "Optimizing location of damage within an enclosed area defined by an algorithm based on the Lamb wave response data", Applied Acoustics 120 ,98-110, 2017

[11] Michele Carboni, Andrea Gianneo, Marco Giglio, «A Lamb waves based statistical approach to structural health monitoring of carbon fibre reinforced polymer composites", Ultrasonics 60 51-64, (2015).

[12] Guoqi Zhao, Ben Wang, Tao Wang, Wenfeng Hao, Ying Luo, “ Detectionand monitoring of delamination in composite laminates using ultrasonic guided wave", composite Structures 225 (2019) 111161.

[13] Zhongqing Su, Lin Ye, "Lamb wave-based quantitative identification of delamination in CF/EP composite structures using artificial neural algorithm », Composite Structures 66, pp 627-637, (2004). 
[14] Zhongqing Su, Lin Ye, Ye Lu, "Guided Lamb waves for identification of damage composite structures: A review", Journal of Sound and Vibration 295 pp 753-780, 2006.

[15] Taoufiq Belhoussine Drissi, Bruno Morvan, Mihai Predoi, Jean-Louis Izbicki, Pascal Pareige, "Study of the transmission of ultrasonic guided wave at the junction of two different elastic plates with the presence of a defect", Key Engineering Materials, pp 21-29, (2011).

[16] Seddiki Mouna, Djelouah Hakim, "Étude de la propagation des ondes de Lamb dans une plaque fissurée", IC-WNDT-MI'14, CSC, Annaba, 09-11 novembre 2014.

[17] Beata Zima, Magdalena Rucka, "Application of wavelet transform in analysis of guided wave propagation signals for damage detection in steel plate" DIAGNOSTYKA, Vol. 16, No. 2 . pp 43-48 (2015).

[18] D. Waltisberg, R. Raišutis, "Group velocity estimation of Lamb waves based on the wavelet transform", ULTRAGARSAS (ULTRASOUND), Vol 63,No 4, pp 35-40, 2008.

[19] Lei Yang, I. Charles Ume, "Inspection of notch depths in thin structures using transmission coefficients of laser-generated Lamb waves", Ultrasonics 63 ,168-173, 2015.

[20] Taoufiq Belhoussine Drissi, Soumaya Zayrit, Benayad Nsiri, Abdelkrim Ammoumou, "Diagnosis of Parkinson's Disease based on Wavelet
Transform and Mel Frequency Cepstral Coefficients", International Journal of Advanced Computer Science and Applications(IJACSA), Volume 10 Issue 3, 2019.

[21] Tsun-Yen Wu, I. Charles Ume, "Fundamental study of laser generation of narrowband Lamb waves using superimposed line sources technique”, NDT\&E International 44,315-323, 2011.

[22] Lei Yang, I. Charles Ume, "Inspection of notch depths in thin structures using transmission coefficients of laser-generated Lamb waves", ultrasonics, Volume 63, December 2015, Pages 168-173.

[23] M. Sifuzzaman, M.R. Islam and M.Z. Ali , "Application of Wavelet Transform and its Advantages Compared to Fourier Transform", Journal of Physical Sciences, Vol. 13, 121-134 (2009).

[24] M. El Allami, H. Rhimini, M. Sidki , "Application of the Complex Mother Wavelet Shan 1-1.5 Processing to Lamb Modes Signals in Plates" International Journal of Science and Research (IJSR), Volume 4, Issue 1, pp 1849-1854, (January 2015).

[25] P. Marical, M. Echcherif Elkettani, M. V. Predoi, «Guides d'ondes a section variable de type Gaussienne, resultats experimentaux et numeriques », GDR US, Giens 14-19 mai 2006.

[26] A. Grossman and J. Morlet, "Decomposition of hardy functions into square integrable wavelets of constant shape", SIAM, pp. 723-726, 1984. 